\title{
A Design of Microstrip Directional Coupler for High Directivity and Tight Coupling
}

\author{
Chul-Soo Kim, Young-Tae Kim, Seung-Hoon Song*, Wan-Soo Jung*, Kwang-Yong Kang** \\ Jun-Seok Park, and Dal Ahn \\ Div. of Information Technology Eng., Soonchunhyang Univ., Chungnam, R.O.Korea \\ *Netel Inc. Seoul, R.O.Korea \\ **Telecommunication Basic Research Lab., ETRI, R.O.Korea \\ E-mail : dahn@ramrec.sch.ac.kr
}

\begin{abstract}
In this paper, we presented a novel structure of microstrip directional coupler for realizing the high directivity characteristic and tight coupling. The achievement of the high directivity with microstrip configuration was carried out by matching the even and odd mode effective phase velocities. By using 2dimensional finite element(FE) calculations, the phase velocity for each mode and design parameters were extracted for given dimensions. Based on the extracted design parameter with phase matched condition, we designed and fabricated $3 \mathrm{~dB}$ and $4.7 \mathrm{~dB}$ directional coupler at $2.0 \mathrm{GHz}$. Experimental results of microstrip coupler show good performance with excellent isolation characteristics.
\end{abstract}

\section{INTRODUCTION}

Directional couplers with parallel microstrip coupled transmission line are widely utilized for various RF and microwave applications because they can be easily incorporated into and implemented with other circuits. However, the microstrip directional couplers suffer from poor directivity due to characteristic of the inhomogeneous dielectric including both dielectric substrate and air in microstrip transmission lines. Thus, the phase velocity of even mode in microstrip is not equal to that of odd mode. The directivity performance of microstrip directional coupler becomes worse when the coupling is decreased or the dielectric permittivity is increased.[1] In addition, it is difficult to achieve tight coupling owing to impractical spacing between the coupled lines in conventional edge coupled microstrip couplers. These are reasons for using the broad-side stripline configuration for tight coupling and high directivity, which needs more fabrication cost and efforts than a conventional microstrip line coupler.
Several techniques are available to equalize or compensate for the inequality in the each mode velocity of the coupled microstrip line. The wiggly-line coupler first proposed by Podell suffers from a lack of pertinent design information.[2] Dielectric overlays have also been used to equalize the mode phase velocities by increasing the odd mode effective dielectric constant.[3] The capacitively and inductively compensated directional couplers with high directivity were used to equalize the phase velocities.[4] Re-entrant mode coupler was proposed by S. B. Cohn to obtain tight coupling.[5]

In this paper, a novel structure of microstrip directional coupler is presented to achieve high directivity and tight coupling, which has $3 \mathrm{~dB}$ coupling and more $30 \mathrm{~dB}$ isolation. To equalize the each mode phase velocity, we used the capacitive compensation method with parallel coupled microstrip line and additional lumped capacitor fabricated using dielectric substrate. The lumped capacitor of the presented structure can be achieved by reducing the difference between the even and odd mode phase velocities for the coupled microstrip line. Based on 2-D finite element(FE) calculations, the phase velocity for each mode and design parameters were extracted for given dimensions. In order to show the effectiveness of newly proposed coupled line structure with additional capacitance, we designed and fabricated a directional coupler to achieve the tight coupling of $3 \mathrm{~dB}$ and $4.7 \mathrm{~dB}$ with high directivity of $32 \mathrm{~dB}$ and $31 \mathrm{~dB}$, respectively.

\section{DESIGN PROCEDURE}

Fig. 1 shows the schematic of the proposed microstrip directional coupler with additional capacitor for tight coupling and high directivity characteristics. This type of proposed coupler is best suited for tight coupling. 


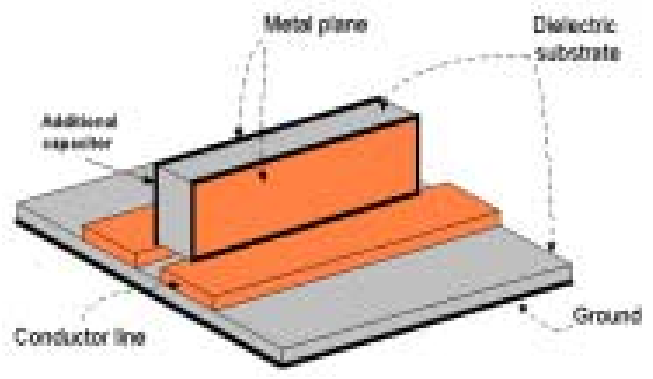

Fig. 1 Schematic of the proposed microstrip directional coupler

Fig. 2 shows the cross sectional view of the proposed microstrip coupler structure for with additional lumped capacitor. Dash line indicates electric wall or magnetic wall when even or odd mode excitations were applied to coupled line of re-entrant mode structure.

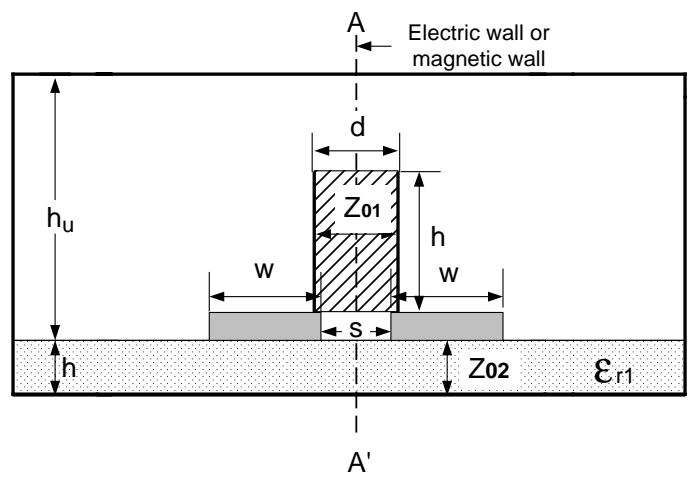

Fig. 2 Cross section view of the proposed microstrip coupler

All parallel-coupled lines, which are supported by any TEM, have the even and odd mode property. The even mode characteristic impedance $Z_{\mathrm{oe}}$ and odd mode characteristic impedance $Z_{o o}$ can be extracted by calculating the characteristic impedance under each mode excitations, respectively. Since the structure can be bisected through its vertical symmetric plane when the even and odd modes are excited as shown Fig. 2, the characteristic impedances of even and odd modes can be derived as follow

$$
\begin{aligned}
Z_{\text {oe }} & =Z_{02} \\
Z_{\text {oo }} & =Z_{02}+2 Z_{01}
\end{aligned}
$$

where $Z_{01}$ means characteristic impedance between additional metal planes above microstrip line, $Z_{02}$ is characteristic impedance between ground plane and microstrip line in Fig 2. In/output matching condition and coupling factor for any directional coupler are given by

$$
\begin{aligned}
& Z_{o}=\sqrt{Z_{o e} Z_{o o}} \\
& k=\frac{Z_{o e}-Z_{o o}}{Z_{o e}+Z_{o o}}=\left|\frac{-Z_{01}}{Z_{02}+Z_{01}}\right| \\
& Z_{o e}=Z_{0} \sqrt{\frac{1+k}{1-k}} \text { and } Z_{o o}=Z_{o} \sqrt{\frac{1-k}{1+k}}
\end{aligned}
$$

where $\mathrm{Z}_{\mathrm{o}}$ and $k$ mean the characteristic impedance and the voltage coupling coefficient, respectively. In the above analysis, it was assumed that the even and odd modes of the coupled line structure have the same velocities of propagation, so that the line has the same electrical length for both modes. For a coupled microstrip, or other nonTEM, this line condition will generally not be satisfied. Generally, the effective permittivity of even mode is greater than that of odd mode in a conventional microstrip coupled line. Thus, the phase velocities for each mode become different. The disagreement of phase velocities gives rise to deterioration in isolation characteristic for conventional microstrip couplers. In order to improve the isolation characteristic in microstrip, the difference in phase velocities should be considered. Fortunately, From Eq.(2) the odd mode impedance $Z_{o o}$, which is related to odd mode phase velocity by Eq.(7), can be adjusted by changing $Z_{01}$, which is determined by the distance between the metal planes and the height shown in Fig.2. It is possible to control odd mode impedance $Z_{\mathrm{oo}}$ and coupling factor $k$ without any effect for $Z_{\text {oe. }}$. Thus, the even and odd mode phase velocity can be well matched.

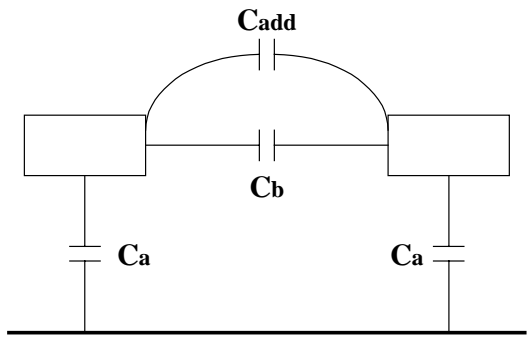

Fig. 3 The equivalent capacitances of proposed structure

Fig 3. shows the equivalent capacitances of presented structure. Capacitance 'Cadd' indicates capacitance between additional metal planes above microstrip line as shown Fig. 2. In the presented equivalent capacitance circuit, we can control the odd mode phase velocity by additional capacitance 'Cadd'. Furthermore 'Cadd' is available to solve the problem for achieving tight coupling owing to impractical spacing between the coupled lines in conventional edge coupled microstrip couplers. 


$$
\begin{aligned}
& v_{p e}=\frac{1}{Z_{o e} C_{a}} \\
& v_{p o}=\frac{1}{Z_{o o}\left(C_{a}+2 C_{b}+2 C_{a d d}\right)}
\end{aligned}
$$

In this paper, design parameters of proposed structure shown in Fig.1 were investigated using numerical calculations based on FEM algorithm. Goals of calculation procedures for the extraction of design parameters are intended to find the acceptable geometric dimensions when the difference of effective permittivities between the even and odd mode become zero. Dielectric constant of substrate for simulations and fabrication was chosen to be 3.05 with 60 mils thick and 2.17 with 10 mil for additional capacitor.

\section{SIMULATION AND MEASUREMENT}

To make even and odd-mode effective permittivity be equal for high directivity, the effective microstrip permittivities are calculated as followings;

$$
\begin{aligned}
& \varepsilon_{e f f e}=\frac{C_{e}}{C_{e 1}} \\
& \varepsilon_{e f f o}=\frac{C_{o}}{C_{o 1}}
\end{aligned}
$$

where the second subscript 1 refers to a free-space ('air') line.

In order to get the capacitance of even and odd mode in microstrip directional coupler as show in Fig. 2, we firstly have to calculate the stored energy within the coupler using FE calculations.[6],[7] Regardless of the dielectric media involved, the stored energy is given by

$$
W=\frac{1}{2} \int \varepsilon_{0} \varepsilon_{r} \nabla u \cdot \nabla u d S
$$

where $\mathcal{E}_{0}, \mathcal{E}_{r}$ are the permittivity of free space, relative permittivity and $u$ means the electric potential. From the energy $W$, the capacitance is then found as

$$
C=\frac{2 W}{(\Delta u)^{2}}
$$

where $\Delta u$ means the potential difference between the two conductors.

The even and odd mode characteristic impedances are then

$$
\begin{aligned}
& Z_{0 e}=\left(c \sqrt{C_{e} C_{e 1}}\right)^{-1}=\frac{\sqrt{\varepsilon_{e f f e}}}{c C_{e}} \\
& Z_{0 o}=\left(c \sqrt{C_{o} C_{o 1}}\right)^{-1}=\frac{\sqrt{\varepsilon_{e f f o}}}{c C_{o}}
\end{aligned}
$$

where $\mathrm{c}$ is the velocity in free space. From Eq. (12) and (13), voltage coupling coefficient factor $k$ can be calculated by using (4). In order to show the validity of this structure for tight coupling and high directivity, microstrip directional coupler with additional capacitor was designed and fabricated in case of coupling value $3 \mathrm{~dB}$ and $4.7 \mathrm{~dB}$. It is very difficult to achieve $3 \mathrm{~dB}$ coupling owing to impractical spacing between the coupled lines in conventional edge coupled microstrip couplers. The presented coupler was implemented by following dimension; 1) in case of $3 \mathrm{~dB}, \mathrm{w}=1.25 \mathrm{~mm}, \mathrm{~s}=0.2 \mathrm{~mm}$, $\mathrm{h}=0.8 \mathrm{~mm}$, and $\mathrm{d}=0.254 \mathrm{~mm}, 2$ ) in case of $4.7 \mathrm{~dB}$, $\mathrm{w}=1.77 \mathrm{~mm}, \mathrm{~s}=0.2 \mathrm{~mm}, \mathrm{~h}=0.5 \mathrm{~mm}$, and $\mathrm{d}=0.254 \mathrm{~mm}$.

Fig. 4 and 5 show simulation results of microstrip coupler with $3 \mathrm{~dB}$ and $4.7 \mathrm{~dB}$ coupling, which was done by Ansoft HFSS, respectively. Simulation results show that the designed directional coupler has the directivity of $29 \mathrm{~dB}$ and $31 \mathrm{~dB}$ at center frequency $2 \mathrm{GHz}$ with excellent matched characteristic. The measured results of fabricated microstrip directional coupler are shown in Fig.6 and 7. Measured results show about $3.15 \mathrm{~dB}$ and $4.87 \mathrm{~dB}$ of coupling, less $30 \mathrm{~dB}$ of return loss, and $32 \mathrm{~dB}$ and $31 \mathrm{~dB}$ of directivity at center frequency $2.0 \mathrm{GHz}$, respectively. Measured performances show excellent agreement with predicted results.

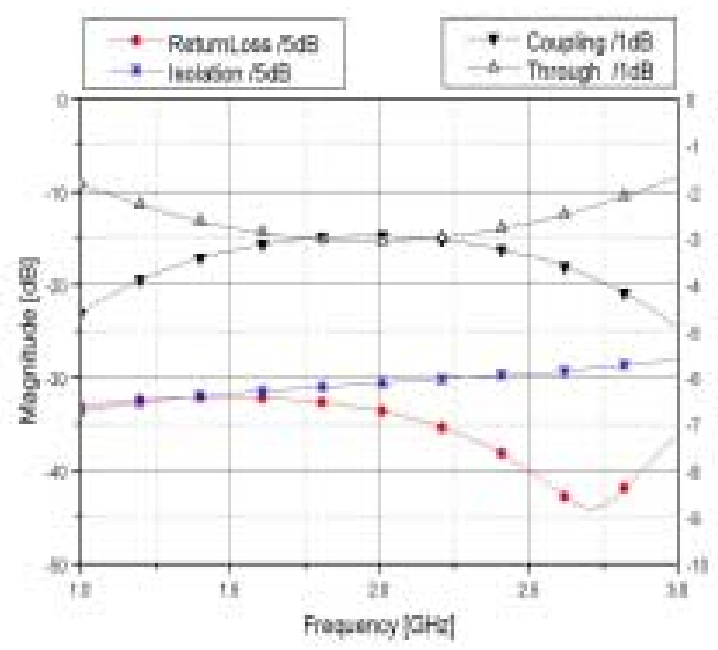

Fig. 4 Simulation result of $3 \mathrm{~dB}$ microstrip coupler 


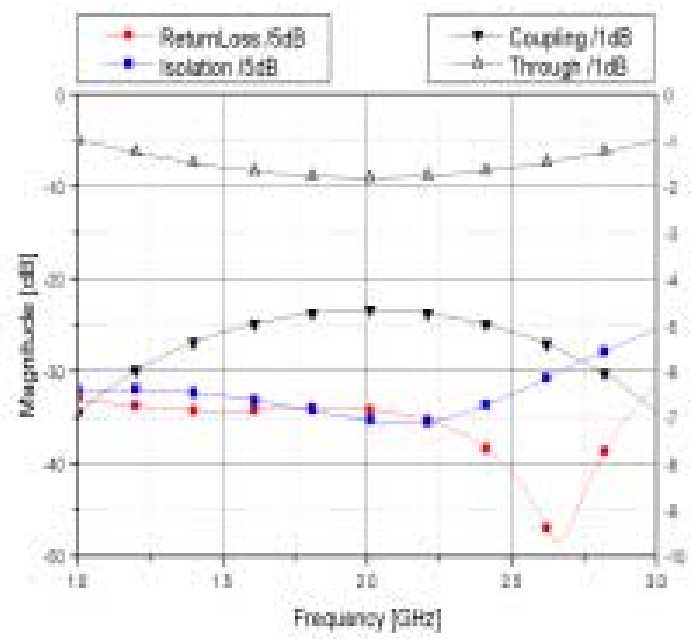

Fig. 5 Simulation result of $4.7 \mathrm{~dB}$ microstrip coupler

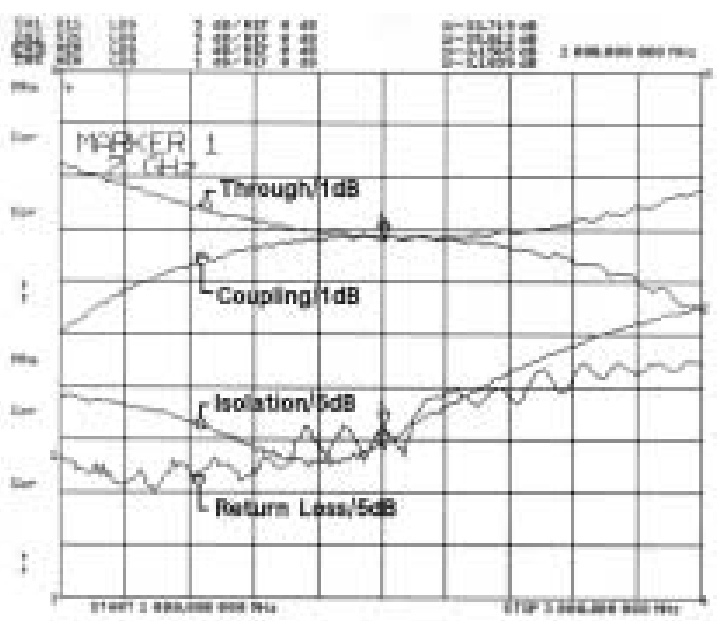

Fig. 6 Measured result of 3dB microstrip coupler

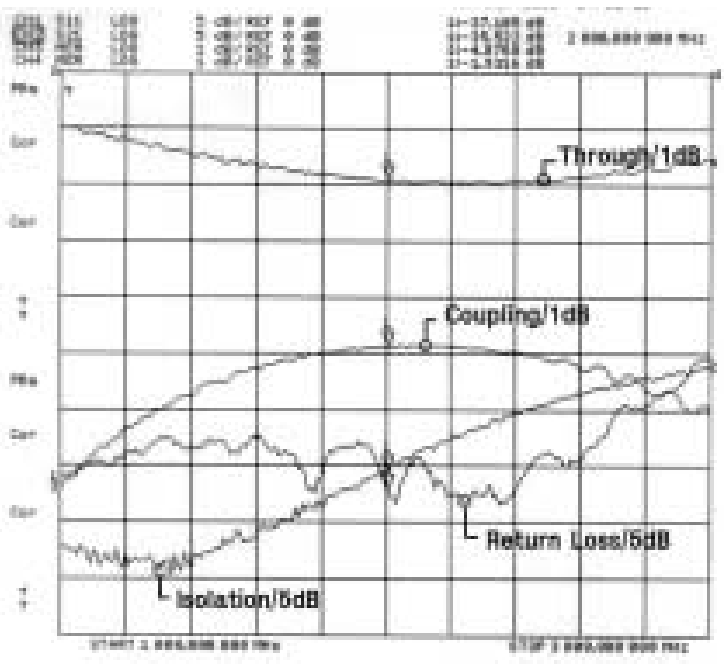

Fig. 7 Measured result of $4.7 \mathrm{~dB}$ microstrip coupler

\section{CONCLUSIONS}

In this paper, we proposed a new structure of microstrip directional coupler with additional capacitor to obtain high directivity and tight coupling characteristics such as $3 \mathrm{~dB}$ coupling. The design procedure is based on the 2-dimensional FE calculations. By changing geometric dimensions of the proposed directional coupler, we can adjust the effective permittivity of odd mode to be equal with that of even mode for the achievement of an identical phase velocity ratio. Experimental results on the fabricated microstrip directional coupler showed the validity of the proposed structure and design procedure. Improvements of poor isolation characteristic and impractical spacing for a conventional microstrip directional coupler with tight coupling can be achieved by using the proposed structure and design procedure

\section{REFERENCE}

[1] Steven L. March, "Phase velocity compen-sation in parallel coupled microstrip," IEEE MTT-S Dig., pp.410-412. 1982.

[2] A. Podell, "A high directivity microstrip coupler technique," IEEE MTT-S Dig., pp.33-56, May 1970.

[3] D. D. Paolino, "MIC overlay coupler design using spectral domain techniques, IEEE Trans. on MTT, vol.26 pp.646-649, Sept. 1978.

[4] M. Dydyk, "Microstrip directional couplers with ideal performance via single-element compensation," IEEE Trans. on MTT, vol.47, No.6, pp.956-964. June 1999.

[5] S. B. Cohn, "The re-entrant cross section and wide-band 3-dB hybrid couplers," IEEE Trans. on MTT, vol. MTT-11, pp.254-258, July 1963.

[6] P. P. Silvester and R. L. Ferrari, Finite Elements for Electrical Engineers: Third Edition. Cambridge University Press, ch.2, 1996.

[7] Nathan Ida and J. P. A. Bastos, Electromagnetics and Calculation of Fields: Second Edition. Springer-Verlag New York, Inc., ch.3, 1997. 